\title{
Review: some but not all topical antimicrobial agents improve the rate of healing of chronic wounds
}

O'Meara SM, Cullum NA, Majid Met al. Systematic review of antimicrobial agents used for chronic wounds. Br J Surg 2001 Jan;88:4-21.

\section{QUESTION: Are systemic and topical antimicrobial agents effective for healing chronic wounds (venous leg ulcers, pressure ulcers, diabetic foot ulcers, and pilonidal sinuses)?}

\section{Data sources}

Studies were identified by searching 19 electronic databases, including Medline and CINAHL, from their inception to January 2000. Conference abstracts, journals, and bibliographies of included studies were handsearched, and experts were contacted.

\section{Study selection}

Randomised controlled trials (RCTs) and nonrandomised trials with a control group were selected if patients had or were at risk for developing diabetic foot ulcers, pressure ulcers, chronic leg ulcers, pilonidal sinuses, non-healing surgical wounds, or chronic cavity wounds; systemic or topical antimicrobial preparations (antibiotic, antifungal, or antiviral agents) intended to prevent or heal wounds were studied; and objective measurement of wound healing, change in skin condition, or development of new lesions was reported.

\section{Data extraction}

Data were extracted on study quality and characteristics, participants, interventions, outcomes, and adverse events.

\section{Main results}

30 trials (25 RCTs) met the inclusion criteria. Trials were grouped according to wound type (table); data were not combined because of study differences. For topical agents for venous ulcers, allopurinol powder, dimethyl sulfoxide powder, silver impregnated charcoal dressing, and tripeptide-copper or silver sulfadiazine improved complete healing of the ulcer area more than placebo or other topical agents. Oxyquinolone ointment improved complete healing of pressure ulcers. Excision with insertion of a gentamicin impregnated sponge and pressure dressing also showed improvement in wound healing for pilonidal sinuses.

\section{Conclusion}

Healing of chronic wounds is improved by some topical antimicrobial agents, but few systemic agents show improvements.

Treatment of chronic wounds or ulcers with systemic antimicrobial or topical agents*

\begin{tabular}{lllll} 
Wound type & Intervention & Studies & Number of patients & Healing \\
Venous ulcers & Systemic agents & 2 RCTs & 84 & No improvement \\
\hline & Topical agents & 5 RCTs, 2 nRCTs & 449 & 3 of 7 improved \\
\hline Mixed causes & Systemic agents & 2 RCTs & 85 & Both improved \\
\hline & Topical agents & 3 RCTs, 2 nRCTs & 128 & 3 of 5 improved \\
\hline Pressure ulcers & Topical agents & 3 RCTs & 169 & 1 of 3 improved \\
\hline Diabetic ulcers & Systemic agents & 2 RCTs & 104 & No improvement \\
\hline & Topical agents & 1 RCT & 29 & Improvement \\
\hline Pilonidal sinuses & Systemic agents & 2 RCTs, 1 nRCT & 190 & 2 of 3 improved \\
\hline & Topical agents & 3 RCTs & 198 & 1 of 3 improved
\end{tabular}

${ }^{*} \mathrm{RCTS}=$ randomised controlled trials; $\mathrm{nRCTs}=$ non-randomised controlled trials.

Source of funding: UK National Health Service.

For correspondence: Dr S M O'Meara, NHS Centre for Reviews and Dissemination,

University of York,

Heslington YO10 5DD,

UK. Fax $+44(0) 1904$ 434556 .

\section{COMMENTARY}

Chronic wounds are a common cause of morbidity, and their cost of care is high. For example, the annual incidence of foot ulcers in patients with diabetes is about $6 \%$, and $16 \%$ of these patients require amputation. ${ }^{1}$ The incidence of pressure sores in a well staffed internal medicine ward was $4 \%{ }^{2}$ The role of infection and colonisation in preventing wound healing is uncertain, and so is the role of antimicrobial agents in promoting recuperation.

The systematic review by O'Meara $e t$ al on antimicrobial agents for treatment of chronic wounds is excellent, with a well defined clinical question, a comprehensive search strategy, and unambiguous criteria for inclusion of studies. The included studies are well described, which allows readers to form their own opinion. The discussion is relevant and comprehensive for both methods and pathophysiological content. Unfortunately, the review offers few recommendations to change clinical practice because little can be learnt from the original studies. Most of them had a small sample size, weak methodology, and unclear biological hypotheses. The abstract cannot do justice to the review by enumeration of positive and negative results. To gain a true insight, the review itself should be read.

Nevertheless, several messages can be highlighted. Overall, systemic antibiotics did not promote healing. However, an effect of antibiotic treatment on healing might have been missed because of a small sample size in some studies. Given the potential to induce resistance, the possible adverse effects, and the lack of evidence for effect, systemic antibiotic treatment should be given only for clear indications of a systemic or local infection.

Topical antimicrobial agents show more promise, but the trial results are inconsistent and the trials have the same methodological problems as do the trials of systemic agents. RCTs of topical antimicrobial agents that use clinically relevant outcomes, a sufficient sample size, and correct methods are urgently needed.

Leonard Leibovici, MD Rabin Medical Center, Petah-Tiqua, Israel

1 Ramsey SD, Newton K, Blough D, et al. Incidence, outcomes, and cost of foot ulcers in patients with diabetes. Diabetes Care 1999;22:382-7.

2 Hagisawa S, Barbenel J. The limits of pressure sore prevention. J Royal Soc Med 1999;92:576-8. 